\title{
Procedimientos heurísticos en las matemáticas de la Antigüedad
}

\author{
Heuristic procedures in ancient Mathematics
}

\author{
Piedad Yuste Leciñena \\ Universidad Nacional de educación a Distancia - UNED*
}

\begin{abstract}
RESUMEN
En este artículo discutiremos los procedimientos griegos de análisis en el contexto de las matemáticas de la Antigua

Babilonia (2000-1600 a.C.), examinado dos ejercicios de contenido muy diferente. Intentaremos probar el método heurístico llevado a cabo en la resolución del problema descrito en la tablilla VAT 8393.

\section{ABSTRACT}

In this paper we will discuss the Greek procedures of analysis in the context of Mesopotamian Mathematics (2000-1600 $B C)$. We try to prove the heuristic method developed in the problem text VAT 8393.

\section{PALABRAS CLAVE}

Matemáticas en Mesopotamia, Geometría Arcaica, Tablilla VAT 8393.

KEYWORDS

Mathematics in the Old Babylonian Period, Ancient Geometry, Tablet VAT 8393.

\section{INTRODUCCIÓN}

Suele decirse que los matemáticos de la Antigua Babilonia (2000-1600 a.C. $\left.{ }^{1}\right)$ fueron expertos en álgebra y poco hábiles en geometría. Estos comentarios se apoyan en el estudio e interpretación de los únicos documentos matemáticos que conocemos hasta ahora: tablas numéricas, listados de unidades y conversiones métricas, ejercicios escolares. Todavía no hemos encontrado ningún texto en el que aparezcan de-

* Departamento de Filosofía de la UNED. Paseo Senda del Rey, 7. 28040 Madrid. E-mail: pyuste@fsof.uned.es

1 La cronología de Mesopotamia se ha realizado en función de los datos astronómicos recogidos en las tablillas, como eclipses lunares y solares, periodos orbitales, etc.; existen cuatro cronologías básicas: baja, media, alta y ultra-alta, cuyo establecimiento se debe a los ciclos registrados en las Tablas de Venus, procedentes del reinado del rey Amişaduqa, nieto de Hammurapi. Los años se ajustan sumando o restando 64 ó 56 años. Ver OPPENHEIM, A. L.; REINER, E: 2003.
\end{abstract}


ducciones ni razonamientos matemáticos; y mucho menos, explicaciones de carácter teórico. Esto se debe a que los maestros de cálculo y geometría seguramente hacían su exposición de modo verbal, trazando diagramas y figuras sobre la arena, inspirándose en la experiencia extraída de las técnicas de agrimensura.

Como veremos más adelante, fue decisivo el papel que desempeñaron las pruebas visuales en todas las culturas de la Antigüedad ${ }^{2}$, no sólo en geometría, que parece bastante obvio, sino también en cierta clase de planteamientos aritméticos en los que intervienen superficies. Para realizar las operaciones básicas de adición, sustracción y multiplicación, los antiguos babilonios utilizaban ábacos y calculi de diversas formas y tamaños ${ }^{3}$. La división se efectuaba mediante el producto por el número inverso. Los alumnos de las escuelas de escribas solían entrenarse en el manejo del cálculo escribiendo y memorizando tablas de multiplicar, de números inversos, de cuadrados y cubos, de raíces cuadradas y cúbicas; además de aprender las unidades métricas en uso y sus respectivas conversiones. Enseñanza muy útil para el futuro desempeño de las funciones administrativas: contabilidad y registro de bienes, cómputo de impuestos, planificación del trabajo de obreros y esclavos, comercios, reparto de herencias, división de terrenos, etc. El sistema de numeración en este período era sexagesimal posicional ${ }^{4}$, con dos signos específicos: uno para expresar la unidad y sus múltiplos y submúltiplos (diš); otro para la decena y sus correspondientes múltiplos (u). No conocían el cero, ni como cifra ni como símbolo que denote la falta de cantidad. Asimismo, disponían de un complejo sistema métrico compuesto de unidades de longitud, superficie, capacidad y peso. Aquí, vamos a utilizar la notación propuesta por Otto Neugenauer:

$$
\ldots x, y, z ; m, n, \ldots_{60} \approx \ldots x \cdot 60^{2}+y \cdot 60+z+m \cdot 60^{-1}+n \cdot 60^{-2} \ldots_{(10}
$$

La sociedad de la que hablamos estaba rígidamente estructurada: un monarca, la corte, funcionarios, sacerdotes, ejército, artesanos, campesinos y esclavos. Los escribas ocupaban un lugar privilegiado en este contexto. Muchos de ellos o ellas ejercían como instructores en los dos niveles básicos de enseñanza: elemental y avanzada ${ }^{5}$. De este segundo estadio proceden algunos de los problemas más complejos que conservamos, aunque, no nos hagamos ilusiones, en ellos sólo aparece el enunciado y una serie de cálculos que conducen al resultado final.

Eminentes investigadores como Otto Neugebauer, François Thureau-Dangin y Evert Bruins ${ }^{6}$ interpretaron muchos de esos cálculos en lenguaje algebraico, porque creyeron descubrir operaciones con potencias, sistemas de ecuaciones y aplicación de reglas formales. Así, identificaron el cuadrado de una magnitud con

2 NETZ, R.: 1999.

3 SCHMAND-BESSERAT, D.:1992. PROUST, Ch.: 2000.

4 Hubo anteriormente otros muchos sistemas de numeración, en los que se combinaban las bases decimal y sexagesimal y los procedimientos de cómputo. Ver NISSEN, H. J.; DAMEROW, P.; ENGLUND, R. K.: 1993. También PROUST, Ch.: 2007 y 2008.

5 PROUST, Ch.: 2007.

6 NEUGEBAUER, O., MKT: 1935-37. THUREAU-DANGIN, F., TMB: 1938. BRUINS, E.; RUTTEN, M., TMS: 1961. 
su potencia; la longitud y anchura de un rectángulo, con las incógnitas a determinar; la superficie de la figura, con el producto de estas últimas. Advirtieron además la semejanza entre el algoritmo que resuelve las ecuaciones de segundo grado y las soluciones propuestas por los escribas, aunque estas vinieran expresadas mediante cantidades específicas.

La primera fase de aproximación a los textos matemáticos babilonios fue una tarea complicada: no sólo había que descifrar los signos grabados en las tablillas de arcilla e interpretar su significado, también era necesario componer un relato coherente y comprobar si las operaciones indicadas conducían al resultado correcto (siempre que la tablilla no estuviera dañada) o detectar posibles errores. No es extraño, por tanto, que Neugebauer y Thureau-Dangin se entusiasmaran al descubrir que muchos de los problemas examinados parecían plantear y resolver ejercicios algebraicos elementales y que, asimismo, los de naturaleza geométrica admitían una lectura algebraica. A estas dificultades hay que añadir el desconocimiento, por aquellos años, de los aspectos culturales y sociológicos que conformaban la civilización babilónica. En el mejor de los casos, se había logrado reconstruir un ejercicio matemático, pero se ignoraba todo acerca de las circunstancias que influyeron en su redacción: quién lo escribió, bajo qué condiciones, en qué lugar, con qué propósito.

Hubo una segunda etapa caracterizada por la traducción masiva de documentos ${ }^{7}$. Pero, a partir de la década de los años 70 del siglo XX, los historiadores de las matemáticas cuidan tanto el examen minucioso de los textos como los aspectos que rodean su elaboración. Haciendo un estudio exhaustivo de los mismos, Jens Høyrup ha descubierto ${ }^{8}$ que algunos de los términos utilizados en la redacción de esos problemas algebraicos, aluden a la construcción de figuras, sensu stricto. Así, llama a estos ejercicios casi algebraicos, pues mientras la línea de argumentación es analítica, el modo de resolución se efectúa dibujando diagramas. Esta interpretación es aceptada hoy en día de modo unánime. Para Jöran Friberg ${ }^{9}$, por ejemplo, las matemáticas de este período componen un álgebra métrica o numérica, que opera con cantidades concretas, y la distingue del álgebra abstracta que maneja especies o magnitudes generales.

No obstante hay dos detalles esenciales que deben ser aclarados. En primer lugar, ¿qué entendemos por análisis? En segundo lugar, ¿qué queremos decir con álgebra? $Y$ ¿en qué sentido ambos procedimientos se relacionan con las matemáticas elaboradas en la antigua Babilonia?

Intentaremos realizar una lectura próxima a los textos, evitando el establecimiento de ecuaciones y la aplicación de reglas abstractas; pues la introducción de herramientas algebraicas distorsiona el significado del mismo, lo desfigura y extrae del entorno en el que fue concebido. Es más, nos traslada a un nivel epistemológico distinto. Debemos ser muy cuidadosos en este sentido, intentar seguir los pasos del escriba, prescindiendo de conjeturas anacrónicas, aunque estas nos per-

\footnotetext{
7 HØYRUP, J: 1996. Aquí analiza las etapas relacionadas con la historiografía mesopotámica.

8 HØYRUP, J: 2002, 20 y ss.

9 FRIBERG, J.: 2007, 2.
} 
mitan alcanzar los resultados correctos de manera más fácil y segura. No se trata de comprobar si el escriba obtenía las soluciones exactas, sino de conocer su metodología, si la hubo y, sobre todo, de comprender el paradigma conceptual en el que moldeó su razonamiento. El resultado de nuestras especulaciones conseguirá levantar barreras entre el procedimiento heurístico llevado a cabo por el escriba y el discurso elaborado por nosotros a fin de obtener una prueba y su generalización. En ocasiones, confundimos ambos argumentos, debido a la intromisión de nuestros propios métodos de investigación.

\section{DEFINICIONES}

Denominamos análisis a la acción de separar las partes de un todo, hasta llegar a conocer sus principios o elementos. También es el proceso cognitivo mediante el cual pasamos de las cosas conocidas y primeramente dadas a otras más generales y desconocidas. Asimismo, es analítica la reducción de un problema a otro más sencillo y fácil de abordar ${ }^{10}$. Análisis, según los matemáticos de la Antigüedad, viene a ser sinónimo de descubrimiento. Proclo atribuyó a Platón la invención del método analítico ${ }^{11}$, el cual consistiría en admitir una opinión unánimemente aceptada, demostrar su falsedad ${ }^{12}$ y sustituirla después por un argumento racionalmente deducido.

El método analítico, según comenta Platón, fue utilizado por primera vez en geometría por Leodamas of $\operatorname{Tasos}^{13}$ (s. IV a.C.), aunque Hipócrates de Quíos (2. ${ }^{a}$ mitad s. V a.C.) ya lo había esgrimido al preparar sus Elementos: Consiste en proponer como hipótesis la verdad que se quiere demostrar y, a partir de ahí, desarrollar un argumento deductivo hasta alcanzar un principio unánimemente aceptado ${ }^{14}$.

Existen dos formas de análisis en geometría:

a) Análisis del Teorema: Se busca comprobar la verdad de un enunciado en una figura determinada. Para ello, se supone que, efectivamente, éste es verdadero. Mediante deducción llegaremos entonces a

1. una proposición admitida como cierta; entonces el enunciado de partida es verdadero

2. una conclusión falsa, entonces el enunciado inicial es falso

10 MAHONEY, M.: 1968, 33. «Reduction itself constituted the foundation of problematical analysis. Confronted with a new problem, the Greek mathematician sought to reduce it to another problem, the solution of which he already knew». HINTIKKA, J.; REMES, U.: 1974. BERGGREN, J. L.; BRUMMELEN, G. VAN: 2000,5 y ss.

11 PROCLUS, In Euclides, 211, 19-22. PLATÓN: República 510 C, 511 A.

12 Menón 98 A. Teeteto 196 E - 197 A. KLEIN, J: 1965.

${ }^{13}$ Filipo de Oponte (ca. 375-347 a.C.) fue alumno de la Academia y autor del Libro XIII de las Leyes o Epinomis; en su opinión, Platón animó a Leodamas a que perfeccionara el método de análisis como forma de demostración en geometría, aunque no podemos atribuirle ningún problema o teorema específico (LASSERRE, F: 1990, 3 y 8).

14 PAPPUS OF ALEXANDRIA, Mathematical Collection VII, 82. También PLATÓN (Menón 86E- 87B). 
b) Análisis del problema: Proporciona la manera de resolver un problema buscando la construcción adecuada. Se actúa como si se conociera la solución. El razonamiento nos llevará entonces a una proposición que

1. si es posible y demostrable por deducción, el problema entonces tendrá una solución determinada

2. si es imposible e indemostrable, no habrá solución

Fuera de la geometría y dentro del contexto de la aritmética, Diofanto ${ }^{15}$ propuso una versión del análisis dirigida a la búsqueda de números concretos: Los datos del problema y la incógnita son trasladados a una igualdad que expresa las condiciones del enunciado. Aplicando dos reglas básicas obtiene el número buscado. Este es el significado que tuvo el álgebra para los matemáticos árabes; el mismo sentido que le dio Vieta In artem analyticam isagoge $(1591)^{16}$.

Algunos de esos problemas algebraicos planteados por los escribas paleobabilonios son actualmente interpretados siguiendo esta misma pauta: representación de las magnitudes conocidas y de la incógnita en un diagrama; manipulación e identificación de las superficies equivalentes; descubrimiento de la magnitud requerida. En cierto modo, esta secuencia es análoga a la propuesta por Diofanto; la diferencia reside en que el matemático alejandrino aplicaba ambas reglas algebraicas ("sustraer lo similar» y «añadir lo que falta») a una relación numérica abstracta, mientras los babilonios lo hacían sobre una estructura geométrica determinada (proceso de «cortar y pegar») ${ }^{17}$.

No obstante, podríamos retroceder a un estadio epistemológico más arcaico en el cual el esquema metodológico sería el siguiente: «Dado el producto (superficie) y la suma (lineal) de dos magnitudes, también son dados los cuadrados de su semidiferencia y de su semisuma. Por tanto, son dadas asimismo las magnitudes buscadas». Aquí, el instructor babilonio traslada su razonamiento a un diagrama (semejante al que encontramos en Euclides, Elementos II.5), mientras los alumnos anotan en sus tablillas cada uno de los pasos realizados, efectuando además las operaciones pertinentes. Las magnitudes dadas poseen el significado que mucho después les concediera Euclides en Data: Son aquellas que pueden ser expresadas y deducidas geométricamente.

El procedimiento aludido nos sirve para resolver los ejercicios algebraicos y los geométricos, pues en todos ellos se extrae el valor de la incógnita a partir de una configuración geométrica concreta. No hay formalización, ni establecimiento de ecuaciones ni reglas que obedecer, salvo las estrictamente geométricas. No obstante, la parte teórica se nos escapa: únicamente podemos adelantar conjeturas e hipótesis acerca de cual fue el tejido de la invención.

15 TANNERY, P.: 1974, Diophantvs Alexandrinvs, Opera Omnia, Stuttgart: Teubner.

16 BALL, W. W. R.: 1960. KLEIN, J: 1968. PARSHALL, K. H.: 1988.

17 Este método de cortar y pegar es ingenuo, porque no discute por qué o bajo qué condiciones son válidas esas transformaciones (HØYRUP, J.: 2007, 128). 


\section{EXAMEN DE UN EJERCICIO ALGEBRAICO}

Los documentos matemáticos procedentes del período paleobabilónico plantean en su mayoría ejercicios aritméticos escolares en los que no se atisba ningún rasgo que apunte a la generalización o formulación teórica. Aunque sí guardan cierto parecido con los estudiados por Diofanto en su Aritmética. Sabemos que el sabio alejandrino enunció las dos reglas básicas del álgebra (semejantes a las descritas por Al-Jwarizmi), trasladando los datos de un enunciado a una expresión formal: El número buscado, o arithmos ${ }^{18}$, junto a los restantes números dados, componen una igualdad con la que se opera hasta conseguir el resultado deseado ${ }^{19}$. El álgebra actúa al servicio del análisis. Proporciona a éste un conjunto de signos, reglas y principios con los que llevar a cabo la investigación. El lenguaje algebraico no tiene necesidad de utilizar modelos geométricos para justificar sus inferencias, aunque a los matemáticos árabes les gustara plasmar en una figura la prueba de sus deducciones.

Veamos un ejemplo clásico tomado de Jens Høyrup ${ }^{20}$ : el problema n. ${ }^{\circ} 12$ contenido en la tablilla BM 13901, registrada en el British Museum. En lenguaje moderno, se supone que se dirime una ecuación bicuadrática.

The surfaces of my two confrontations I have accumulated: 0;21,40. My confrontations I have made hold: 0;10. The moiety of 0;21,40 you break: 0;10,50 and 0;10,50 you make hold, 0;1,57,21 +25$\}, 40$ is it. 0;10 and $0 ; 10$ you make hold, 0;1,40 inside 0;1,57,21\{+25\},40 you tear out: by $0 ; 0,17\{+25\}, 40,0 ; 4,10$ to one 0;10,50 you append: by $0 ; 15,0 ; 30$ is equalside. $0 ; 30$ the first confrontation. 0;4,10 inside the second 0;10,50 you tear out: by 0;6,40, 0;20 is equalside. 0;20 the second confrontation.

El maestro de cálculo dicta en primera persona las condiciones del problema. Luego instruye a sus alumnos acerca de cómo resolverlo. Los babilonios no utilizaron letras ni signos para expresar las magnitudes; ya fueran estas conocidas o desconocidas, las identificaron siempre por su nombre o por su cantidad; si esta no era conocida, recurrían al método de falsa posición, es decir, le asignaban un valor acordado o la unidad. Nosotros las nombraremos con letras para reconocerlas de una manera más inmediata y precisa, o para dar una apariencia general a los razonamientos.

A) Datos del ejercicio o magnitudes dadas:

1. Superficie acumulada (suma) de dos cuadrados $(S+Q): 0 ; 21,40$

2. Superficie sustentada (producto) por los lados de ambos cuadrados $(s \times q): 0 ; 10$

\footnotetext{
18 La incógnita o arithmos (número) es representada siempre por un signo.

19 CHRISTIANIDIS, J.: 2007.

20 HØYRUP, J.: 2002, 71-77.
} 
B) Construcción del diagrama:

1. Rectángulo Principal:

a) Longitud dada: $(S+Q)=0 ; 21,40$

b) Anchura desconocida: $(Q)$

2. Cuadrado desconocido: $(s \times q)$

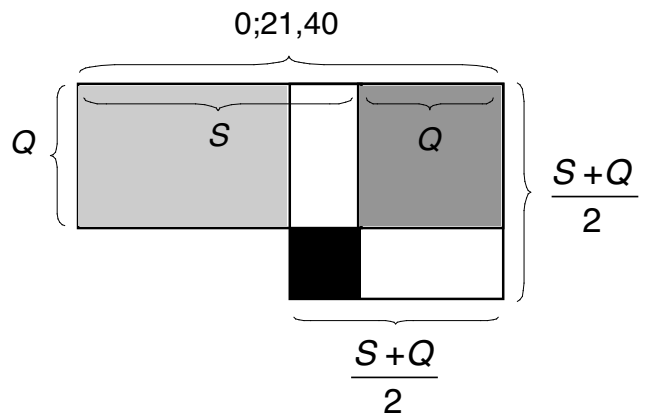

Figura 1. Jens Høyrup (2002, p. 73).

C) Sucesivas magnitudes dadas:

1. Semisuma de la longitud: $\frac{1}{2}(S+Q)=0 ; 30 \times 0 ; 21,40=0 ; 10,5$

2. Su cuadrado: $0 ; 10,50 \times 0 ; 10,50=0 ; 1,57,21,40$

3. Superficie del rectángulo secundario:

$S \times Q=(s \times q) \times(s \times q)=0,10 \times 0 ; 10=0 ; 1,40$

4. Cuadrado complementario: $0 ; 1,57,21,40-0 ; 1,40=0 ; 0,17,21,40$

5. Su lado: $\sqrt{0 ; 0,17,21,40}=0 ; 4,10$

6. Valor de $Q: 0 ; 10,50+0 ; 4,10=0 ; 15$

7. Valor de $S: 0 ; 10,50-0 ; 4,10=0 ; 6,40$

8. Medida del lado $q: \sqrt{0 ; 15}=0 ; 30$

9. Medida del lado $s: \sqrt{0 ; 6,40}=0 ; 20$

Høyrup llama la atención acerca del carácter figurativo de las operaciones aritméticas realizadas. Así, dos segmentos se enfrentan uno a otro para componer un cuadrado. Las superficies se acumulan cuando se suman. Los rectángulos se forman sosteniendo la longitud sobre la anchura. Como los ejercicios escolares de este período no contienen diagramas, debemos suponer que fueron los instructo- 
res quienes los dibujaban sobre tableros de polvo o arena mientras explicaban el método a seguir ${ }^{21}$.

Euclides en Elementos II.5, probará las relaciones que configuran el diagrama anterior.

\section{REVISIÓN DE UN EJERCICIO GEOMÉTRICO}

El problema planteado en VAT 8393 representa un caso único en la geometría de la Antigüedad ${ }^{22}$. Procede de un lugar indeterminado del período paleobabilónico, posiblemente de la mítica ciudad de Nippur. El texto incluye solamente el enunciado, los cálculos aritméticos y la figura de un trapecio isósceles, subdividido en cinco trapecios menores, con sus respectivas diagonales y valores numéricos. En esta sección nos limitaremos a seguir las instrucciones del escriba.

\section{A) Enunciado propuesto}

Dado un trapecio simétrico (isósceles) y su diagonal, encontrar otros cuatro trapecios isósceles —dos a la izquierda (superiores) y dos a la derecha (inferiores) - enlazados sucesivamente y cuyas diagonales respectivas tengan la misma longitud ${ }^{23}$.

Jöran Friberg rectifica la figura dibujada de la tablilla utilizando las medidas proporcionales:

39503730

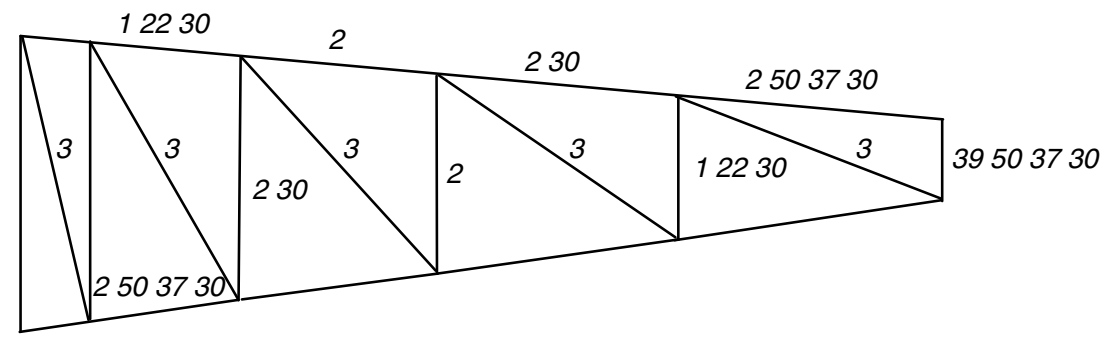

Figura 2. Jöran Friberg ${ }^{24}$.

21 En YBC 8633 el alumno dibuja en su tablilla una figura que justifica la metodología aplicada (YUSTE, P.: 2008).

22 Tabilla conservada en el Vorderasiatisches Museum of Berlin; publicada y estudiada por Jöran Friberg y Joachim Marzahn; también por YUSTE, P.: 2009. Friberg comenta: «No similar constructions appear to be known from any other mathematical documents, Greek, or Islamic, or whatever.» (FRIBERG, J: 2007, 440).

${ }^{23}$ La unidad de longitud es el «n i n d a», equivalente a $6 \mathrm{~m}$. El UŠ es su inmediata superior, equivalente a $360 \mathrm{~m}$.

${ }^{24}$ Esta no es la figura que aparece en la tablilla, sino la propuesta por Friberg, más adecuada a las proporciones indicadas en el texto $(2007,431)$. 
B) Datos del ejercicio: Los lados del trapecio (T) y su diagonal

- Frente superior ( $F$ ): 2;30 UŠ (150 $\mathrm{n}$ i n d a, en notación decimal)

- Frente inferior $(f): 2$ UŠ (120 $\mathrm{n}$ i $\mathrm{n}$ d a, en notación decimal)

— Longitud ( $L$ ): 2 UŠ (120 $\mathrm{n}$ i n d a, en notación decimal)

— Diagonal (d): 3 UŠ (180 $\mathrm{n}$ in d a, en notación decimal)

Dibujamos la Figura 3 para comprender mejor las operaciones realizadas en la tablilla. El uso de letras y expresiones simbólicas evita que los resultados obtenidos se atribuyan exclusivamente a las propiedades internas de los números. Como veremos después, el escriba apoyará su deducción en la siguiente relación [1], no justificada: $\frac{F-f}{L}=\frac{l_{1}-L}{f}=k$.

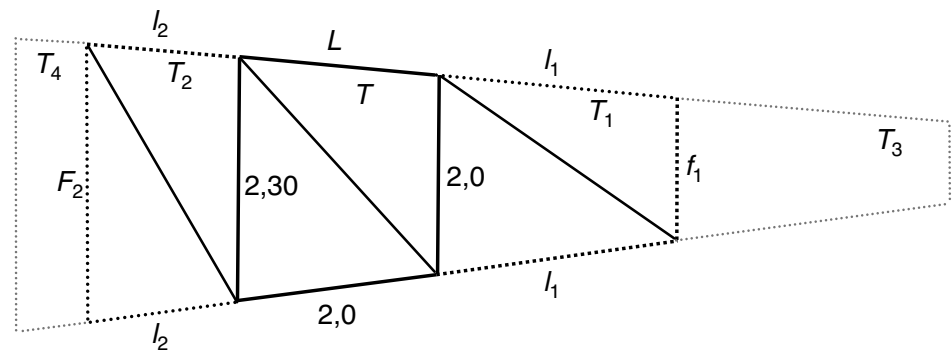

Figura 3.

C) Búsqueda del primer trapecio inferior $\left(T_{1}\right)$

Situado inmediatamente a la derecha del primero. Se conoce, entonces su frente superior: 2 UŠ

- Cálculo del ninda-nu o factor de proporcionalidad (lo llamamos k) ${ }^{25}$ :

$$
2 ; 30-2=0 ; 30 \rightarrow 0 ; 30 \times \frac{1}{2}=0 ; 15 \rightarrow k=0 ; 15 \text { (decimal: 0,25) }
$$

- Cálculo de la longitud del nuevo trapecio:

$$
2 \times 0 ; 15=0 ; 30 \rightarrow 2+0 ; 30=2 ; 30
$$

- Cálculo del frente inferior del nuevo trapecio:

$$
2 ; 30 \times 0 ; 15=0 ; 37,30 \rightarrow 2-0 ; 37,30=1 ; 22,30
$$

${ }^{25}$ Factor que Jöran Friberg denomina «the growth rate» $(2007,439)$. 


\section{D) Búsqueda del primer trapecio superior}

Situado inmediatamente a la izquierda del primer trapecio. Se conoce, entonces su frente inferior: 2;30 UŠ.

- Cálculo de la longitud:

$$
2 ; 30 \times 0 ; 15=0 ; 37,30 \rightarrow 2-0 ; 37,30=1 ; 22,30
$$

- Cálculo del frente superior:

$$
0 ; 15 \times 1 ; 22,30=0 ; 20,37,30 \rightarrow 2 ; 30+0 ; 20,37,30=2 ; 50,37,30
$$

E) Búsqueda de los restantes trapecios

El escriba realiza operaciones análogas para calcular las medidas de las otras dos figuras.

- Segundo trapecio inferior:

Frente superior dado: $1 ; 22,30$

Longitud: $2 ; 30+1 ; 22,30 \times 0 ; 15=2 ; 50,37,30$

Frente inferior: $1 ; 22,30-2 ; 50,37,30 \times 0 ; 15=0 ; 39,50,37,30$

- Segundo trapecio superior:

Frente inferior dada: 2;50,37,30

Longitud: $1 ; 22,30-2 ; 50,37,30 \times 0 ; 15=0 ; 39,50,37,30$

Frente superior: $2 ; 50,37,30+0 ; 15 \times 0 ; 39,50,37,30=3,0 ; 35,9,22,30$

\section{EXPLICACIÓN GEOMÉTRICA}

Los cálculos anteriormente indicados responden a un plan que no se muestra en la tablilla. En los apartados sucesivos intentaremos reconstruir el razonamiento del maestro.

\section{A) Cálculo del factor de proporcionalidad}

El coeficiente $k$, en un trapecio simétrico, expresa la relación entre la diferencia de los frentes y su longitud: $\frac{F-f}{L}=k$. Este factor es esencial en la determinación de los lados desconocidos de las figuras trapezoidales y lo encontramos en numerosos ejercicios procedentes de este período. Suele aludir a una conversión ge- 
ométrica que, en este caso, no es necesario realizar ${ }^{26}$. Jöran Friberg lo denomina «the growth rate» ${ }^{27}$, indicando la propiedad que conservan los triángulos semejantes.

\section{B) Deducción del primer trapecio inferior}

La relación [1]: $\frac{F-f}{L}=\frac{I_{1}-L}{f}=k$, esencial en la determinación de la longitud $\left(I_{1}\right)$, nos proporciona la pista sobre la posibilidad de que el instructor esté dibujando un trapecio auxiliar, $T^{\prime}$, como vemos en la figura 4a. Además, el escriba advierte en la tablilla que esta primera longitud $\left(l_{1}\right)$ se obtiene «multiplicando el ninda- $n u(k)$ por la longitud inferior (2)...», en lugar de haber escrito: «por el frente inferior (también 2)...», tal y como se deduce de la expresión [1], escrita numéricamente en la tablilla. Este detalle nos induce a pensar que la longitud inferior aludida pertenece al lado de una figura $\left(T^{\prime}\right)$ no mencionada en el texto.

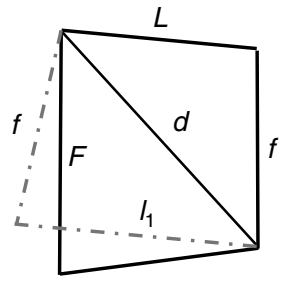

Figura 4a.

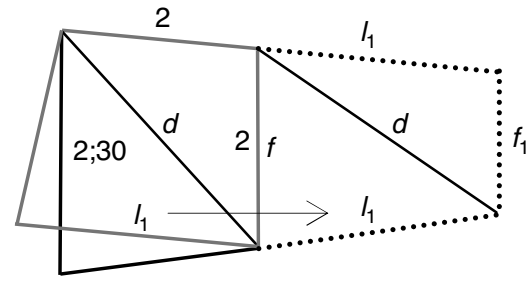

Figura $4 b$.

El argumento que acompaña al diagrama sería el siguiente:

Dado el trapecio simétrico $T$, entonces, también será dado otro trapecio, $T^{\prime}$, cuya longitud $(f)$, frente inferior $(L)$ y diagonal son comunes (Figura $4 a$ ):

- El cálculo de la longitud, $I_{1}$, viene determinado por la relación [1]:

$$
k=\frac{2 ; 30-2}{2}=\frac{l_{1}-2}{2} \rightarrow I_{1}=2 ; 30
$$

El paso de $T^{\prime}$ a $T_{1}$ se sustenta en que ambos trapecios contienen el mismo triángulo $\left(f, d, l_{1}\right)$. No obstante, se puede demostrar aplicando la teoría de semejanza de triángulos.

\footnotetext{
${ }^{26}$ Se trata de ejercicios que aplican la formula del agrimensor para determinar la superficie de un trapecio irregular: producto de los promedios de los lados opuestos. A pesar de esta inexactitud, la operación de convertir la figura dada en un trapecio recto, inscrito en un cuadrado, permitió resolver muchos de estos ejercicios. HØYRUP, J.: 2002.YUSTE, P.: 2006.

27 FRIBERG, J.: 2007, 439.
} 
- Dado $T^{\prime}$, también será dado $T_{1}$ (Figura 4b); ambos trapecios, $T$ y $T_{1}$ cumplen la siguiente relación: $\frac{F-f}{L}=\frac{f-f_{1}}{l_{1}}=k$

$$
k=\frac{2 ; 30-2}{2}=\frac{2-f_{1}}{2 ; 30} \rightarrow f_{1}=1 ; 22,30
$$

\section{C) Deducción del primer trapecio superior}

La explicación es análoga a la anterior. El escriba dibuja un trapecio simétrico, $T$ ", en el que coinciden el frente superior, la longitud y la diagonal (Figura 5). Asimismo, establece la relación [1] para ambos.

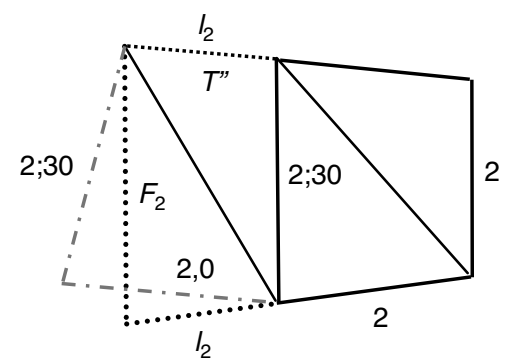

Figura 5.

- El cálculo de la longitud, $I_{2}$, viene determinado por la siguiente expresión:

$$
k=\frac{2 ; 30-2}{2}=\frac{2-I_{1}}{2 ; 30} \rightarrow I_{2}=1 ; 22,30
$$

- Dado $T^{\prime \prime}$, también será dado $T_{2}$; porque se cumple:

$$
k=\frac{2 ; 30-2}{2}=\frac{F_{2}-2 ; 30}{1 ; 22,30} \rightarrow F_{2}=2 ; 50,37,30
$$

\section{SECUENCIA COMPLETA}

Aplicando este procedimiento sucesivamente, se logra componer una cadena de trapecios isósceles $\left\{T_{4}, T_{2}, T, T_{1}, T_{3}\right\}$ con diagonales de la misma longitud (Figura 6). Sin embargo, obtenemos además otra secuencia de trapecios, simétrica de la anterior, al reunir y ordenar (Figura 7) todas las figuras punteadas $\left\{T^{\mathrm{iv}}, T^{\prime \prime}, T^{\prime}\right.$, $\left.T^{\prime \prime \prime}, T^{\vee}\right\}$, cuyas longitudes respectivas corresponden a los frentes de los trapecios de la Figura 6, y viceversa. 
A) Primera secuencia

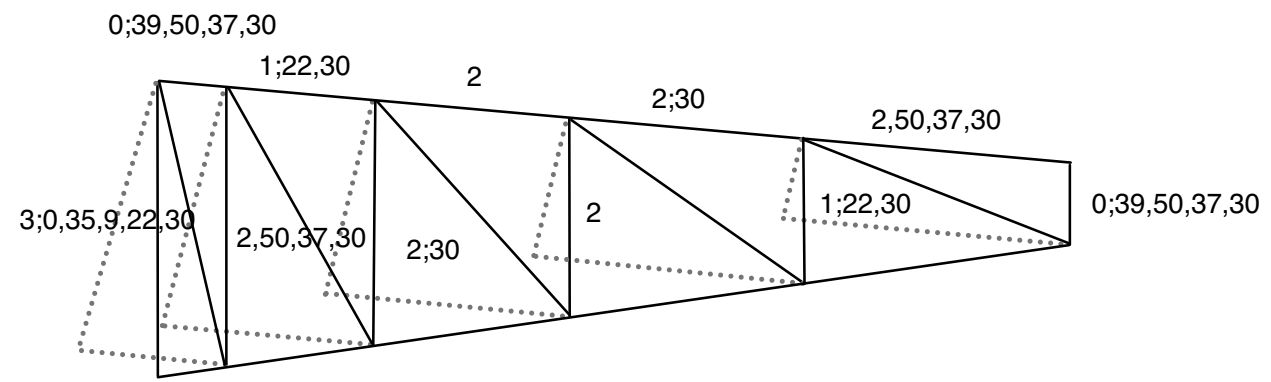

Figura 6.

\section{B) Secuencia simétrica}

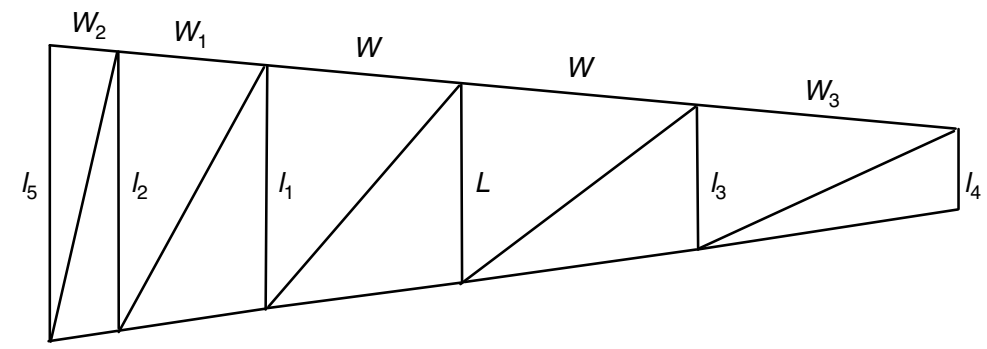

Figura 7.

Esta construcción plantea varias interrogantes: ¿su resultado es casual? ¿Existiría la misma simetría e identidad si hubiéramos escogido magnitudes diferentes? ¿Podemos generalizar este argumento? ¿Cómo sabemos que el trapecio $T$ ' y los restantes de esta colección cumplen la relación [1]? Los escribas babilonios no conceptualizaron la noción de ángulo, pero sí poseían una idea intuitiva de la igualdad de triángulos. Pudieron advertir que la proporción existente entre la diferencia de los frentes respecto de la longitud es invariante para todos los trapecios de ambas colecciones.

No obstante, Jöran Friberg ${ }^{28}$ ha demostrado algebraicamente que dicha expresión es la condición necesaria que deben cumplir todos los trapecios encadenados que tienen la misma medida de la diagonal. Para ello, introduce la regla de la diagonal (o teorema de Pitágoras), disponible en este período, y establece un sistema de ecuaciones.

28 Jöran Friberg formaliza esta deducción sintética (2007, 440). 


\section{CONCLUSIÓN}

El propósito de este trabajo ha consistido, principalmente, en averiguar el proceso heurístico llevado a cabo en la resolución de un ejercicio geométrico concreto: el descrito en VAT 8393. Para ello, hemos tomado los instrumentos conceptuales disponibles en la época que estudiamos, evitando realizar planteamientos algebraicos anacrónicos, como son el establecimiento de ecuaciones, aislamiento de las incógnitas, aplicación de reglas formales, composición de sistemas de ecuaciones, etc.

En mi opinión, la metodología expuesta aquí nos aproxima al llamado contexto del descubrimiento; mientras la reconstrucción algebraica propuesta por Jöran Friberg nos sitúa más bien en el contexto de la justificación. Observamos también en qué sentido las nociones de análisis y álgebra difieren: si el análisis propone la incógnita como magnitud dada y evoluciona a partir de este dato, el álgebra estructura y ordena; introduce normas y directrices a seguir.

El ejercicio VAT 8393 representa un caso concreto, en el que todas las magnitudes de la primera figura poseen su respectiva imagen en la segunda. Además, esta simetría aparece incluso dentro de la misma composición, pues a cada una de las longitudes corresponde un frente gemelo. El historiador se pregunta entonces si este ejercicio trata un caso excepcional y único, determinado por las especiales medidas de los lados del primer trapecio o, por el contrario, es uno más en su especie. El modo de averiguarlo consiste en aplicar el método sintético, como hace Jöran Friberg: demostrar que la relación [1], $\frac{F-f}{L}=\frac{l_{1}-L}{f}=k$, descubierta por el escriba, es condición necesaria para obtener una secuencia de trapecios con la misma medida de la diagonal. Y para ello, conjuga dos proposiciones básicas: la regla de Pitágoras y el algoritmo que expresa la proporcionalidad entre los lados de dos trapecios isósceles consecutivos: $\frac{F-f}{L}=\frac{f-f_{1}}{l_{1}}=k$; concluyendo con el escriba que la relación que determina la diferencia entre las longitudes de dos trapecios consecutivos, respecto de su frente común, es la misma que la existente entre la diferencia de los frentes, y la respectiva longitud, para cualquiera de ellos.

\section{BIBLIOGRAFÍA}

BALL, W. W. R. (1960). A short account of the history of mathematics, New York: Dover Publications.

BERGGREN, J. L.; BRUMMELEN, G. van (2000): «The Role and development of Geometric Analysis and Synthesis in Ancient Greece and Medieval Islam», in SUPPES, etc., pp. 1-31.

BRUINS, E. M.; RUTTEN, M. (1961). Textes mathématiques de Suse (TMS). Mémoires de la Mission archéologique en Iran, XXXIV, Paris.

CHRISTIANIDIS, J. (2007). «The way of Diophantus: Some clarifications on Diophantus' method of solution», Historia Mathematica 34, pp. 289-305. 
|04_Piedad.qxp 8/1/10 08:23 Página 66 